\title{
Metabolic engineering of Rhodotorula toruloides IFO0880 improves C16 and C18 fatty alcohol production from synthetic media
}

\author{
J. Carl Schultz ${ }^{1,2}$, Shekhar Mishra ${ }^{1,2}$, Emily Gaither ${ }^{1,2}$, Andrea Mejia' , Hoang Dinh ${ }^{2,3}$, Costas Maranas ${ }^{2,3}$ and \\ Huimin Zhao ${ }^{1,2,4^{*}}$ (1)
}

\begin{abstract}
Background: The oleaginous, carotenogenic yeast Rhodotorula toruloides has been increasingly explored as a platform organism for the production of terpenoids and fatty acid derivatives. Fatty alcohols, a fatty acid derivative widely used in the production of detergents and surfactants, can be produced microbially with the expression of a heterologous fatty acyl-CoA reductase. Due to its high lipid production, $R$. toruloides has high potential for fatty alcohol production, and in this study several metabolic engineering approaches were investigated to improve the titer of this product.
\end{abstract}

Results: Fatty acyl-CoA reductase from Marinobacter aqueolei was co-expressed with SpCas9 in R. toruloides IFO0880 and a panel of gene overexpressions and Cas9-mediated gene deletions were explored to increase the fatty alcohol production. Two overexpression targets ( $A C L 1$ and ACC1, improving cytosolic acetyl-CoA and malonyl-CoA production, respectively) and two deletion targets (the acyltransferases $D G A 1$ and $L R O 1$ ) resulted in significant (1.8 to 4.4-fold) increases to the fatty alcohol titer in culture tubes. Combinatorial exploration of these modifications in bioreactor fermentation culminated in a $3.7 \mathrm{~g} / L$ fatty alcohol titer in the $L R O 1 \triangle$ mutant. As $L R O 1$ deletion was not found to be beneficial for fatty alcohol production in other yeasts, a lipidomic comparison of the DGA1 and LRO1 knockout mutants was performed, finding that $D G A 1$ is the primary acyltransferase responsible for triacylglyceride production in $R$. toruloides, while $L R O 1$ disruption simultaneously improved fatty alcohol production, increased diacylglyceride and triacylglyceride production, and increased glucose consumption.

Conclusions: The fatty alcohol titer of fatty acyl-CoA reductase-expressing $R$. toruloides was significantly improved through the deletion of $\angle R O 1$, or the deletion of DGA1 combined with overexpression of $A C C 1$ and $A C L 1$. Disruption of $L R O 1$ surprisingly increased both lipid and fatty alcohol production, creating a possible avenue for future study of the lipid metabolism of this yeast.

Keywords: Rhodotorula toruloides, Fatty alcohols, Metabolic engineering, Lipidomics, CRISPR/Cas9

\footnotetext{
${ }^{*}$ Correspondence: zhao5@illinois.edu

1 Department of Chemical and Biomolecular Engineering, Carl R. Woese Institute for Genomic Biology, University of Illinois at Urbana-Champaign, Urbana, IL 61801, USA

Full list of author information is available at the end of the article
}

\section{Background}

Long-chain alcohols are widely used as constituents of cosmetics, surfactants, and detergents [1]. These compounds have traditionally been produced chemically, through hydrolysis and hydrogenation of plant or animal fats, or directly synthesized by oligomerization of ethylene followed by oxidation of the resulting olefin [2]. In the United States, synthesis from ethylene is the original author(s) and the source, provide a link to the Creative Commons licence, and indicate if changes were made. The images or other third party material in this article are included in the article's Creative Commons licence, unless indicated otherwise in a credit line to the material. If material is not included in the article's Creative Commons licence and your intended use is not permitted by statutory regulation or exceeds the permitted use, you will need to obtain permission directly from the copyright holder. To view a copy of this licence, visit http://creativecommons.org/licenses/by/4.0/. The Creative Commons Public Domain Dedication waiver (http://creativeco mmons.org/publicdomain/zero/1.0/) applies to the data made available in this article, unless otherwise stated in a credit line to the data. 
predominant method [2]; however, this process requires the use of non-renewable petroleum feedstocks as well as the highly energy-intensive production of ethylene by steam cracking at $750-950{ }^{\circ} \mathrm{C}$ [3]. The use of microbes as cellular factories for chemical conversion has been increasingly investigated for many chemical products including fuels [4], pharmaceuticals [5], and various other value-added bioproducts [6] from renewable feedstocks at approximately ambient temperature and pressure, creating the potential for significant energy savings and reduced carbon emissions compared to traditional chemical synthesis approaches.

Fatty alcohols are natively produced in many organisms as a component of natural waxes by reduction of fatty acids or fatty acyl-CoA by carboxylic acid reductases (CARs) or fatty acyl-CoA reductases (FARs), respectively [1]. These fatty alcohol-producing genes have been expressed in a variety of heterologous microbial hosts including Escherichia coli [7], Synechocystis species [8], Saccharomyces cerevisiae, Yarrowia lipolytica [9], and Rhodotorula toruloides [10], enabling fatty alcohol production by microbial fermentation. Strikingly, fatty alcohol titers and yields remain far below those obtained for related compounds such as lipids and fatty acids $[11,12]$. Significant metabolic rewiring of genetically tractable organisms such as E. coli and S. cerevisiae has led to noteworthy increases in the fatty alcohol titers obtained, with E. coli producing $6.3 \mathrm{~g} / \mathrm{L}$ with the deletion of thioesterase gene tes $C B$, lactate dehydrogenase gene $l d h A$, acetate kinase gene $a c k A$, and phosphate acetyltransferase gene pta [13]. S. cerevisiae produced $6.0 \mathrm{~g} / \mathrm{L}$ of fatty alcohols using FAR from Mus musculus after 9 distinct genome edits were combined including targets for deletion and overexpression, and replacing the negative regulator of the GAL1 promoter used for FAR expression with a positive regulator [14]. Oleaginous yeasts such as Y. lipolytica and $R$. toruloides are promising candidate hosts for fatty alcohol bioproduction owing to their high levels of acylCoA production, which is natively used to produce lipids. However, metabolic engineering efforts in these less domesticated yeasts are less sophisticated, with Y. lipolytica producing $5.8 \mathrm{~g} / \mathrm{L}$ of fatty alcohols using FAR from Marinobacter aquaeolei (MaFAR) after overexpression of $D G A 1$, mutagenesis of the Mga2 regulator, and expression of 2 copies of FAR [9]. Although production of $8 \mathrm{~g} / \mathrm{L}$ fatty alcohols has been reported from growth of $R$. toruloides on YP-sucrose media in a fed-batch fermentation also using MaFAR [10], no metabolic engineering efforts towards production of fatty alcohols have been reported to date, likely due to the lack of advanced gene editing tools compared to E. coli and S. cerevisiae. Nevertheless, the impressive results achieved so far in oleaginous yeasts demonstrate their potential as production hosts for fatty acid derivatives. With additional metabolic engineering work, the fatty alcohol titers produced in these yeast species can doubtlessly be further increased.

Recently, the genetic engineering toolkit for $R$. toruloides has been improved significantly with development of CRISPR gene editing [15-17] and RNA interference [18], and characterization of many promoters [19-21]. In this study, we applied the CRISPR/Cas9 gene editing system we previously developed in combination with overexpression of native and heterologous genes to create and explore a broad selection of fatty alcohol-producing upregulation and knockout mutants in $R$. toruloides IFO0880. We observed that improving the supply of precursor metabolites, blocking the formation of TAGs, and disrupting genes potentially involved in fatty alcohol degradation all improved the fatty alcohol titer of the producing strain. Beneficial modifications were then explored combinatorially at the culture tube and bioreactor scale, and lipidomic analysis of engineered strains shed light on the differing roles of the acyltransferases $D G A 1$ and LRO1 in the lipid metabolism of $R$. toruloides.

\section{Results \\ Selection of promoter and FAR gene for fatty alcohol production}

Various FAR genes have been tested for production of fatty alcohols in different yeast species. D'Espaux and coworkers compared the previously used Marinobacter aqueaolei VT8, Tyto alba, and Mus musculus FARs in S. cerevisiae, finding that MmFAR1 yielded the highest titer [14], while in Yarrowia lipolytica, Cordova and coworkers compared FAR genes from Apis mellifera, Homo sapiens, Arabidopsis thaliana, and M. aqueaolei, finding that FAR from M. aqueaolei (maqu_2220 or MaFAR) showed the best performance [9]. In $R$. toruloides, FARs from $M$. aqueaolei, T. alba, A. thaliana, A. mellifera, G. gallus, and $A$. domesticus were recently compared, with MaFAR also giving the highest titer in this species [22]. Owing to its high activity in both $R$. toruloides and Y. lipolytica, MaFAR was selected for use in this study. It is possible this enzyme functions particularly well in oleaginous yeasts as its preferred substrates are the highly plentiful C16 and C18 acyl-CoA [23].

The GAPDH, or $T D H 3$, promoter is known to be a strong promoter in the model yeast $S$. cerevisiae and the native version has frequently been used for heterologous gene expression in $R$. toruloides [10, 24, 25]. While a recent study of constitutive promoters in $R$. toruloides identified various promoters stronger than $p G A P D H$ such as the transcription elongation factor promoter ( $p T E F 1)$ and the adenine nucleotide transporter promoter (pANT1) in a GFP expression assay, when pANT1 and $p G A P D H$ were compared for fatty alcohol production in 
another recent study in $R$. toruloides, $p G A P D H$-driven expression resulted in a four-fold greater titer of $50 \mathrm{mg} / \mathrm{L}$ $[19,22]$. 1-hexadecanol and 1-octadecenol constituted approximately $25 \%$ each of the total fatty alcohols, while the remaining $50 \%$ were 1 -octadecanol. We compared pTEF1- and $p G A P D H$-driven MaFAR expression and similarly found $p G A P D H$ to give a higher titer of fatty alcohols (Fig. 1). Therefore, $p G A P D H$ was used to drive MaFAR expression in all subsequent experiments.

\section{Optimization of CRISPR/Cas9 gene editing in $R$. toruloides IFO0880}

The creation of knockout strains has long been an essential step for the metabolic engineering of microbial organisms, typically with the goal of preventing carbon flux from traveling through undesirable pathways, increasing the flux available for the intended product [1]. Homologous recombination (HR)-based gene disruption was previously used in $R$. toruloides to delete PEX10 with the goal of improving lipid production [12]. Due to the very low efficiency of HR in the wild-type $R$. toruloides, the nonhomologous end-joining (NHEJ)-associated gene KU70 was first disrupted. Subsequently an antibiotic marker with $1 \mathrm{~kb}$ homology arms targeting the PEX10 locus was transformed and PEX10 was disrupted with $27 \%$ efficiency.

CRISPR/Cas9-mediated gene editing is advantageous compared to HR-based strategies in that only a $20 \mathrm{bp}$ guide RNA (gRNA) must be cloned rather than $1 \mathrm{~kb}$

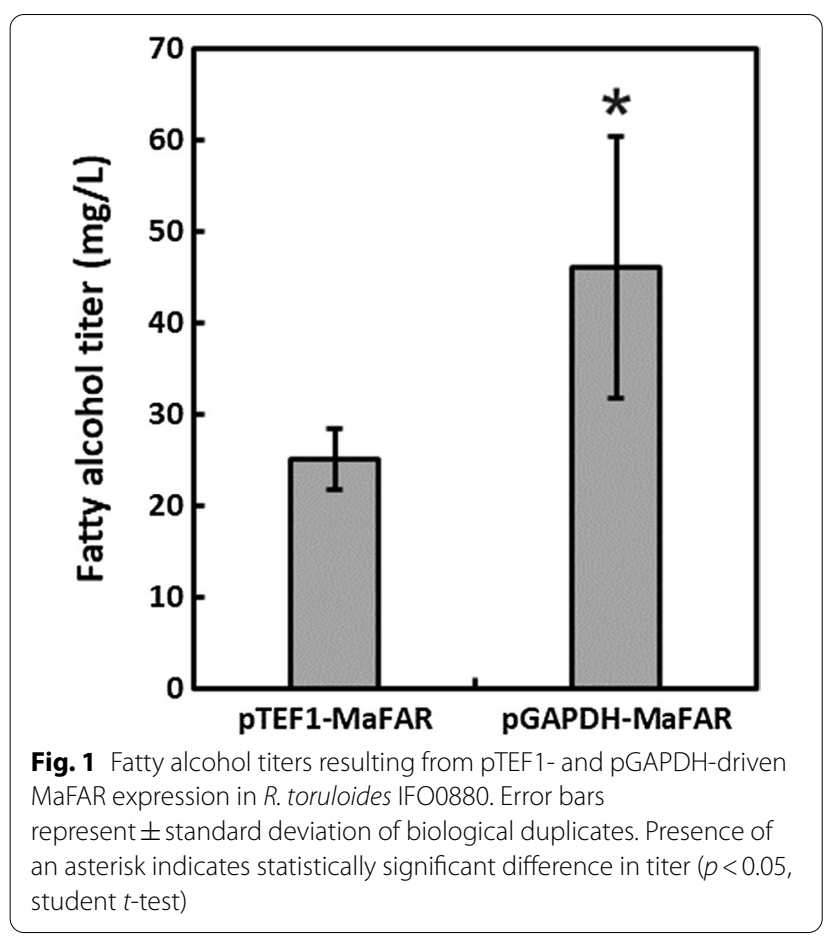

homology arms, and this editing can be performed in a wild-type $R$. toruloides strain rather than a KU70-deficient strain, which is known to suffer from much lower transformation efficiency [12]. We previously reported CRISPR/Cas9 gene editing in the $R$. toruloides strain NP11 with $>95 \%$ efficiency in the deletion of the carotenogenic reporter gene CAR2 [15], with pPGK1-driven SpCas9 expression and gRNA expression driven by a fusion 5S rRNA-tRNA ${ }^{\text {Arg }}$ promoter. We attempted to directly transfer this system into $R$. toruloides IFO0880, which has become the better characterized strain due to various tool development, omics and modeling studies $[19,26-28]$, but found there was no gene editing activity. The PGK1 promoter elements of the strains NP11 and IFO0880 are $92 \%$ similar and the two strains overall share $95 \%$ genetic similarity [25]. However, our results suggest the strains are dissimilar enough that promoter elements are not always interchangeable between them.

CRISPR/Cas9-mediated gene editing was also previously reported in the $R$. toruloides strain IFO0880, using genetic elements from this strain, with $p G A P D H$-driven SpCas9 expression and tRNA ${ }^{\mathrm{Tyr}}$-driven gRNA expression, albeit with a lower efficiency of $46 \%$ in the deletion of CAR2 [16]. Reasoning that the problem with our design was related to the use of promoter elements from NP11, we next attempted to improve the efficiency of our system by testing a variety of medium ( $p G A P D H, p 27)$ and strong ( $p T E F 1, p A N T 1$, and $p 17$, driving expression of an unknown gene [19]) promoters driving SpCas9 expression and either tRNA ${ }^{\text {Tyr }}$ or 5S rRNA-tRNA ${ }^{\text {Tyr }}$ driven gRNA expression in the knockout of CAR2. We first randomly integrated the Cas9 expression cassette to IFO0880, then selected two colonies from each Cas9 transformation to receive the gRNA expression cassette subsequently. In contrast to FAR expression, Cas9 gene editing activity benefitted substantially from replacing $p G A P D H$ with stronger promoters such as $p T E F 1$, $p A N T 1$, and $p 17$, while the medium strength $p 27$ resulted in similar efficiency as $p G A P D H$ (Table 1 ). In most cases both gRNA expression systems performed similarly. The combination of pANT1 and 5S-tRNA ${ }^{\mathrm{Tyr}}$ showed $100 \%$ editing efficiency in both colonies screened and was chosen to drive Cas9 and gRNA expression, respectively, for subsequent metabolic engineering efforts. The sequences for the codon-optimized SpCas9, MaFAR, and all other synthetic genes used in this study are shown in Additional file 1: Table S1.

\section{Metabolic engineering to improve fatty alcohol production in $R$. toruloides}

To create a platform strain for our engineering efforts, a combined $p$ GAPDH-MaFAR and $p A N T 1-S p C a s 9$ expression cassette was randomly integrated to the $R$. toruloides 
Table 1 CAR2 disruption efficiency obtained from combinatorial optimization of Cas9 and gRNA promoters for high-efficiency gene disruption, screening 2 random Cas 9 integrants per Cas 9 promoter tested

\begin{tabular}{lcl}
\hline & tRNA $^{\text {Tyr }}(\%)$ & 5S-tRNA $^{\text {Tyr }}$ \\
\hline pGAPDH-SpCas9 & 2 & n.d \\
p27-SpCas9 & 13 & \\
& 13 & $17 \%$ \\
pTEF1-SpCas9 & 9 & $10 \%$ \\
pANT1-SpCas9 & 93 & $97 \%$ \\
& 96 & $85 \%$ \\
p17-SpCas9 & 0 & $100 \%$ \\
& 2 & $100 \%$ \\
\end{tabular}

IFO0880 genome. Random integration mutants were screened for FAR activity, and Cas9 activity in the deletion of $C A R 2$ as reported previously $[14,15]$, and a clone (subsequently referred to as $880 \mathrm{CF}$ ) was isolated with $99 \%+$ efficiency for CAR2 deletion upon transformation of a CAR2-targeting gRNA and producing a fatty alcohol titer of $50 \mathrm{mg} / \mathrm{L}$ in glass culture tubes with a $10 \%$ dodecane overlay. Subsequent engineering was performed by randomly integrating either a gRNA expression cassette targeting the beginning of an ORF for NHEJ-mediated gene disruption, or a $p 17$-driven overexpression cassette to $880 \mathrm{CF}$. G418 was used as the selection marker for the combined Cas9 and MaFAR integration, while hygromycin and nourseothricin were used for gRNA and overexpression cassette integrations, respectively. A schematic overview of the strain engineering process used in this study is provided in Fig. 2. To mitigate the issue of variable expression resulting from random integration, three colonies were screened for each overexpression target, the fatty alcohol titers were measured and the clone with the highest titer (corresponding to the most optimal expression level for beneficial overexpression targets) was preserved and tested in replicate for the final comparison. For deletions, genomic DNA at the target locus (a predicted high-efficiency cut site in the first 5-10\% of the gene ORF) was sequenced and clones containing a frame-shift mutation were considered to have the target gene disrupted. gRNAs used, their gene deletion efficiencies, and the specific gene mutations observed are described in Additional file 1: Table S2.

Many genetic targets and strategies have been explored for the metabolic engineering of different yeast species for fatty alcohol production. For the engineering of $R$. toruloides, we tested various approaches that have shown prior success in yeast including upregulating the production of precursor metabolites, blocking pathways that compete for metabolic flux, reactivating acyl chains sequestered in lipids, and blocking fatty alcohol degradation pathways [9, 14] (Fig. 3). For overexpression targets, the native genomic copy of a gene was used unless otherwise noted. Mycocosm proteinIDs are provided for each native gene targeted in Additional file 1: Table S3 and a full list of strains created in this study is provided in Additional file 1: Table S4. The strains created were compared in glass culture tubes containing synthetic complete media with a $10 \%$ dodecane overlay inoculated to an initial OD600 of 0.1. The fatty alcohol concentration in the overlay was measured by GC-FID after 6 days of growth (Fig. 4).

Increasing production of precursor metabolites: MaFAR produces fatty alcohols through the reduction of fatty acyl-CoA chains, consuming two molecules of NADPH per fatty alcohol molecule produced [23].

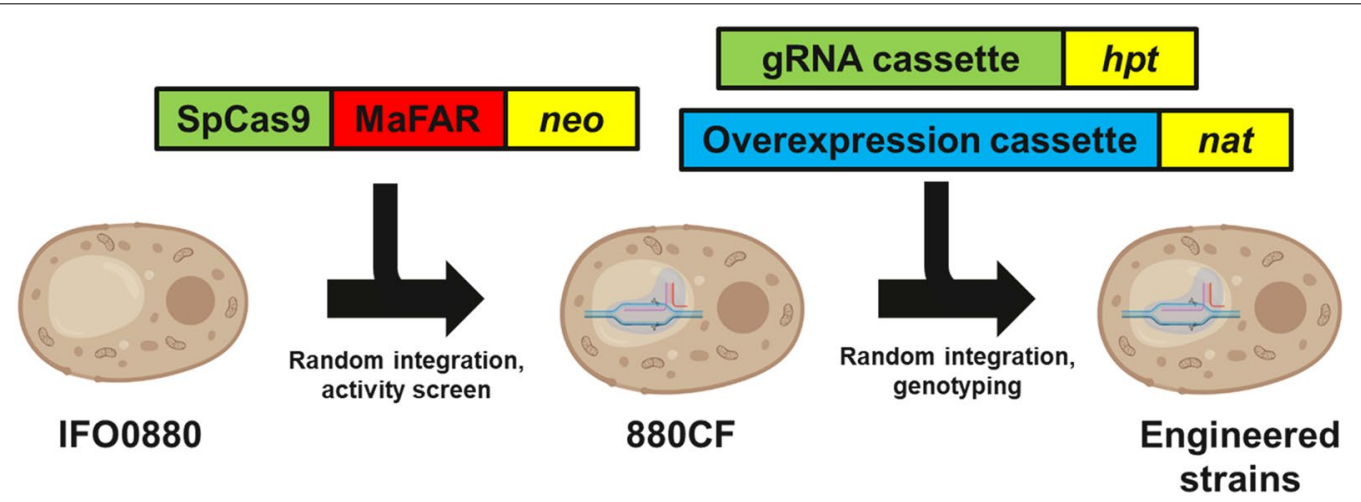

Fig. 2 Schematic overview of the strain engineering process employed in this study. SpCas 9 and MaFAR were co-integrated to create a platform strain, 880CF, followed by integration of gRNA cassettes to create knockout mutants and gene overexpression cassettes for overexpression strains. neo gene encoding aminoglycoside 3'-phosphotransferase (G418 resistance), hpt gene encoding hygromycin phosphotransferase (hygromycin resistance), nat gene encoding nourseothricin acetyltransferase (nourseothricin resistance) 


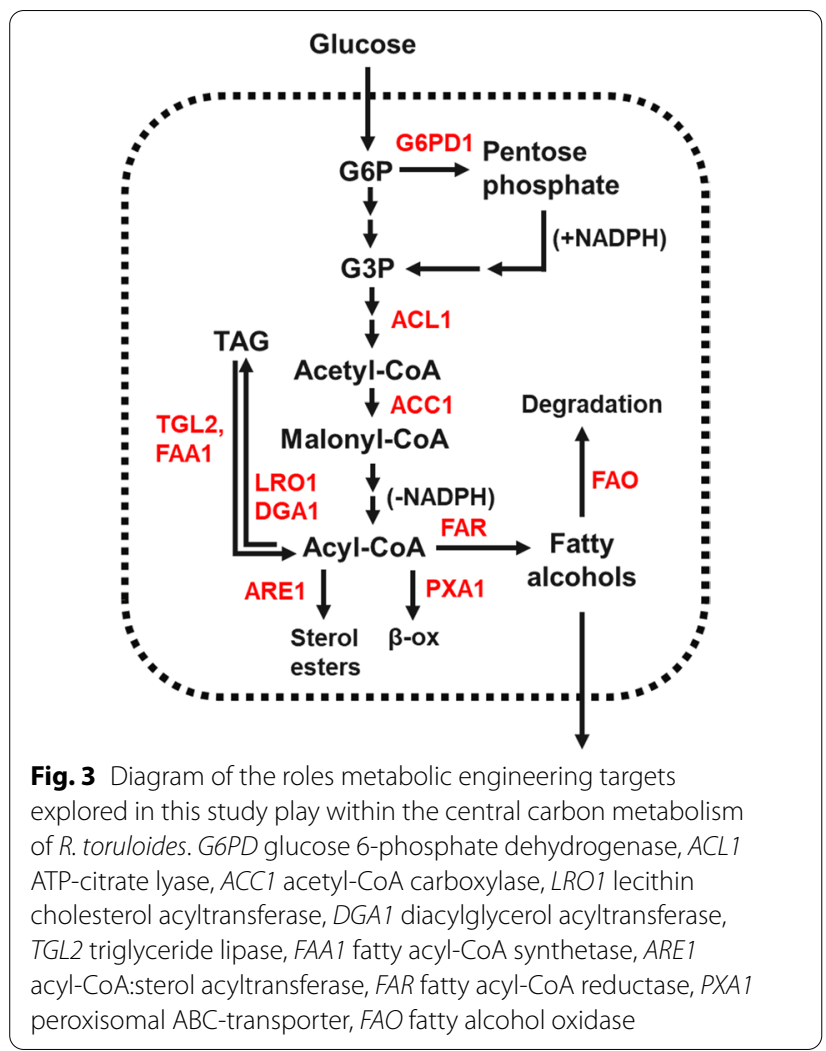

Fatty acyl-CoA chains are produced by the fatty acid synthase complex, consuming one malonyl-CoA molecule and two NADPH molecules per two-carbon extension of the growing acyl-CoA chain [1]. Therefore, we tested overexpression of the native $A C L 1$ to increase cytosolic acetyl-CoA production, overexpression of the native $A C C 1$ to increase malonyl-CoA production from acetyl-CoA, and overexpression of three NADPH-producing genes $(R$. toruloides glucose-6-phosphate dehydrogenase gene (G6PD1), Y. lipolytica G6PD1, and $Y$. lipolytica malic enzyme gene (ME1)).

$A C C 1$ and $A C L 1$ overexpression resulted in notable 1.8-fold and 3.7-fold increases in the fatty alcohol titer, respectively. Overexpression of the native G6PD1 or $Y$. lipolytica G6PD1 and $M E 1$ did not significantly increase the titer.

Blocking or reversing lipid formation: In wild-type $R$. toruloides, as in other oleaginous yeasts, the typical destination for fatty acyl-CoA chains is storage in lipid droplets as triacylglycerides (TAGs) [29]. We attempted to block this competing pathway through the disruption of two genes involved in TAG formation, DGA1 and LRO1. DGA1 catalyzes the transfer of an acyl chain from an acyl-CoA molecule to a diacylglyceride (DAG), while $L R O 1$ forms TAGs by transferring an acyl chain from a phospholipid to a DAG [30]. We additionally tested the disruption of ARE1 encoding an acyltransferase involved in sterol esterification [31].

Disruption of DGA1 and LRO1 increased the fatty alcohol titer by 2.3-fold and 4.4-fold, respectively, indicating that these acyltransferases (or in the case of $L R O 1$, possibly an upstream protein as acyl-CoA is not thought to be a direct substrate) are noteworthy competitors with MaFAR for acyl-CoA molecules. ARE1 disruption, however, did not increase the fatty alcohol titer. Due to the promising results of $D G A 1$ and LRO1 disruption, simultaneous knockout of both genes was attempted by integrating a single construct with expression cassettes for gRNAs targeting both genes and sequencing the resulting colonies at both gene loci. However, only single knockouts, and a small number of very slow-growing colonies unable to be sequenced were obtained, suggesting the DGA1 $L$ LO1 $\triangle$ double mutation may be lethal, or strongly detrimental to growth in $R$. toruloides. As these two genes are expected to account for the majority of TAG formation [30], one possible explanation for the failure to obtain the double mutant is that the inability to form TAGs confers a severe fitness defect in $R$. toruloides.

An alternative approach we explored was to reactivate acyl chains that have been sequestered in TAGs, first by hydrolyzing TAGs with triglyceride lipase (TGL2) to form free fatty acids, then reactivating the free fatty acids with acyl-CoA synthetase (FAA1). As overexpression of both genes may be necessary for this strategy to work, FAA1 and TGL2 overexpression cassettes driven by the $A N T 1$ and TEF1 promoters, respectively, were cloned to a single plasmid and integrated simultaneously. However, overexpression of these two genes did not increase the fatty alcohol titer.

Blocking fatty alcohol degradation: The elimination of fatty alcohol degradation pathways has been shown to improve fatty alcohol titers in S. cerevisiae and Y. lipolytica $[9,14]$. Three IFO0880 genes were identified with homology to $Y$. lipolytica fatty alcohol oxidase gene (FAO1). Furthermore, a popular strategy for increasing lipid production which has also proved effective in improving fatty alcohol titers is to disrupt $\beta$-oxidation, either by disrupting peroxisome biogenesis through deletion of PEX10 [32, 33], or by interfering with peroxisomal uptake of fatty alcohols or fatty acyl-CoA by deleting the peroxisomal transporter genes PXA1 and PXA2 [14]. While PEX10 is reported to be essential in $R$. toruloides [27] (and supporting this, we were unable to obtain any viable $P E X 10 \triangle$ mutants), two genes were identified with homology to S. cerevisiae PXA1 and PXA2 and successfully targeted for disruption. However, disruption of the three FAO genes and both PXA1-like genes did not significantly improve the fatty alcohol titer. 


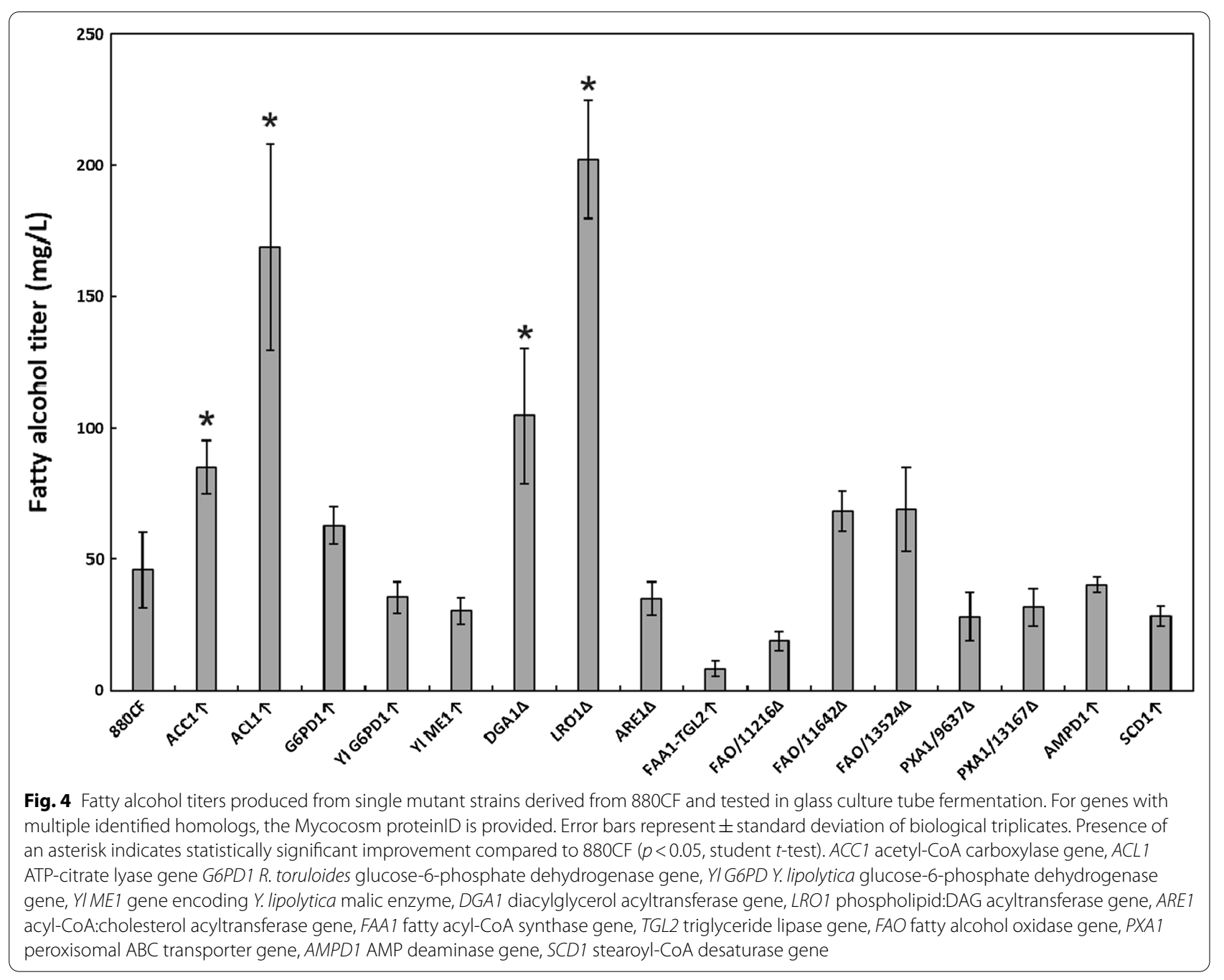

Other targets: Overexpression of stearoyl-CoA desaturase gene (SCD1 or OLE1) [34] and AMP deaminase gene $(A M P D 1)$ [33] have both been shown to increase lipid production in $Y$. lipolytica, while SCD1 overexpression also improved fatty alcohol production in $S$. cerevisiae [14]. AMPD1 is involved in the initiation of lipid biosynthesis, reducing the mitochondrial AMP concentration to inhibit isocitrate dehydrogenase, resulting in the accumulation of citrate, which can be converted to cytosolic acetyl-CoA by ACL1 following export from the mitochondria [35]. SCD1 converts stearoyl-CoA to oleyl-CoA, relieving stearoyl-CoA-mediated allosteric inhibition of $\mathrm{ACC} 1$, allowing for more malonyl-CoA formation in the presence of long-chain acyl-CoA molecules [34]. However, we found that neither of these approaches increased the fatty alcohol titer in $R$. toruloides.

Combinatorial exploration of validated single targets: Although we were unable to disrupt the two most promising deletion targets, $L R O 1$ and DGA1, in the same strain, both single knockouts were evaluated in combination with the two best overexpression targets, ACC1 and $A C L 1$, individually and in tandem (Fig. 5). Combination of DGA1D with $A C C 1$ overexpression was found to increase the fatty alcohol titer from the initial $106 \mathrm{mg} / \mathrm{L}$ to $206 \mathrm{mg} / \mathrm{L}$. However, all of the LRO1D strains with $A C C 1$ or $A C L 1$ overexpression had lower fatty alcohol titers than the starting strain's titer of $202 \mathrm{mg} / \mathrm{L}$.

\section{Bioreactor fermentation of fatty alcohols}

Three of the best-performing $D G A 1 \triangle$-based strains

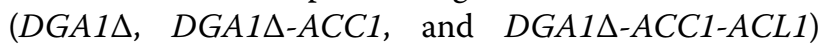
and $L R O 1 \triangle$-based strains (LRO1 $L R O 1 \triangle-A C L 1$, and $L R O 1 \triangle-A C C 1-A C L 1)$ were tested in $250 \mathrm{~mL}$ Eppendorf DASbox Mini Bioreactors with a $20 \%$ dodecane overlay in week-long fed-batch fermentations with glucose concentrations restored to $50 \mathrm{~g} / \mathrm{L}$ each day for the first five days (Fig. 6, Table 2). As in the culture tubescale tests, the $L R O 1 \Delta$ strain performed better than its 


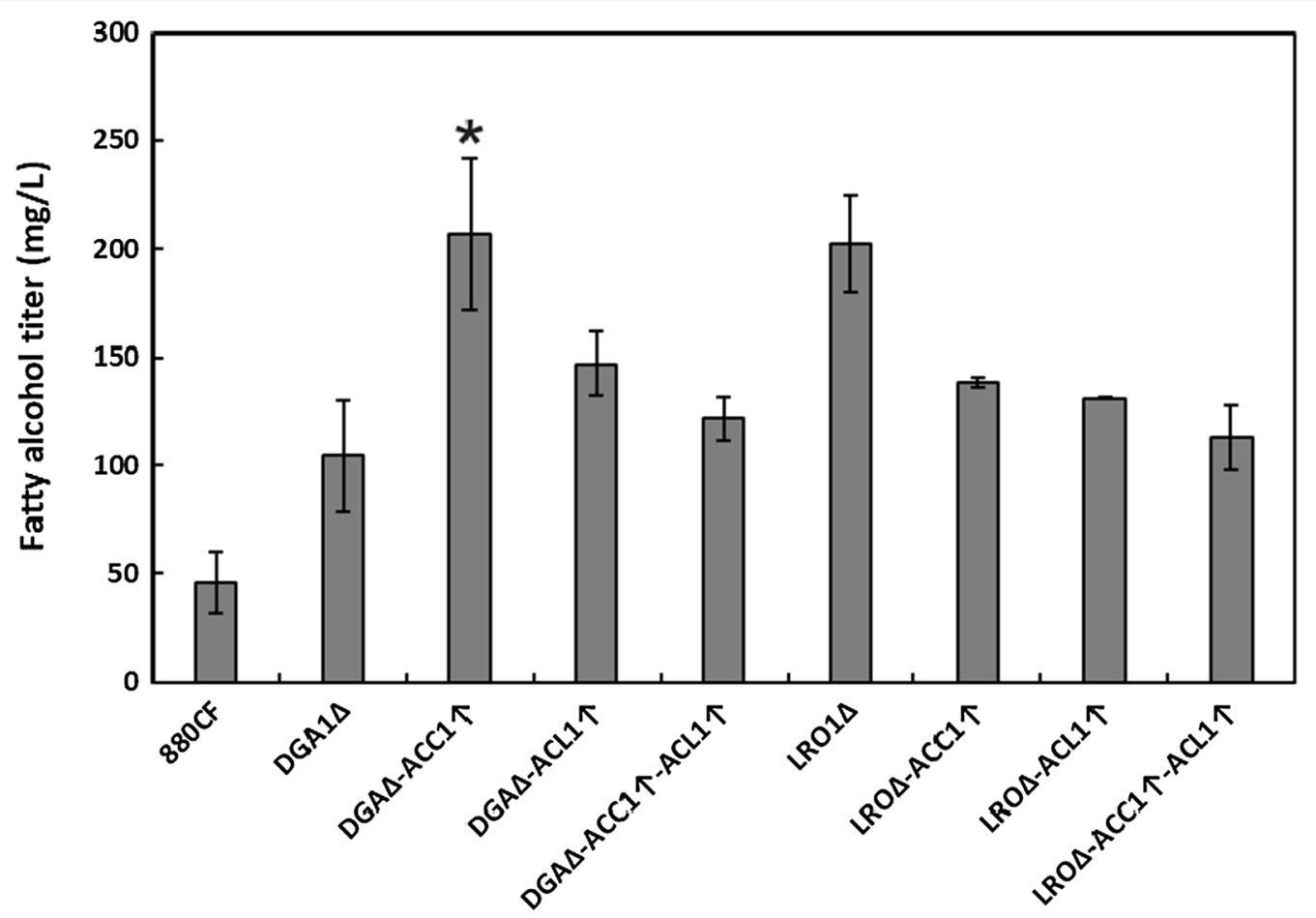

Fig. 5 Fatty alcohol titers produced from double and triple mutant strains derived from 880CF and tested in glass culture tube fermentations. Error bars represent \pm standard deviation of biological triplicates. Presence of an asterisk indicates statistically significant improvement compared to the parent strain ( $p<0.05$, student $t$-test). ACC1 acetyl-CoA carboxylase gene, ACL1 ATP-citrate lyase gene, DGA1 diacylglycerol acyltransferase gene, LRO1 phospholipid:DAG acyltransferase gene

derivatives with additional overexpression targets, while the DGA1 $\triangle$ strain benefitted from $A C C 1$ and $A C C 1$ $A C L 1$ overexpression. The highest fatty alcohol titer and yield of $3.7 \mathrm{~g} / \mathrm{L}$ and $0.024 \mathrm{~g} / \mathrm{g}$ glucose, respectively, were obtained in 880CF-LRO1 $\triangle$. In each experiment, the peak fatty alcohol titer was reached no later than day 7 . Each strain showed similar growth characteristics, reaching an OD600 between 25 and 30 after approximately $48 \mathrm{~h}$ of growth. In most cases, major fatty alcohol secretion began at this point and continued until $\sim 48 \mathrm{~h}$ after the glucose feed was stopped.

\section{Lipidomic analysis of DGA1 1 and $L R O 1 \triangle$ Rhodotorula strains}

Our finding that $L R O 1 \triangle$ improves fatty alcohol production more than $D G A 1 \triangle$ in $R$. toruloides stands in contrast to a report in S. cerevisiae, where DGA1 deletion was highly beneficial but $L R O 1$ deletion abolished fatty alcohol production entirely [14]. It is surprising that $L R O 1 \Delta$ would improve fatty alcohol production so significantly, as the reaction LRO1 is known to catalyze (reversible transfer of an acyl chain from a phospholipid to a DAG) does not directly use acyl-CoA as a substrate, unlike
DGA1 which consumes acyl-CoA to convert DAGs into TAGs.

To investigate and compare the mechanisms by which these knockouts lead to improved fatty alcohol production in $R$. toruloides, a lipidomic study was performed comparing the strains 880CF, 880CF-LRO1A, and 880 CF-DGA1 $\triangle$ after six days of growth on synthetic media. Most strikingly, the DGA1D mutant has greatly reduced TAG accumulation, as expected, while the LRO1 $\triangle$ mutant has increased TAG and DAG accumulation (Fig. 7a, b). This result suggests that DGA1 is likely a net TAG producer and direct competitor with MaFAR for acyl-CoA molecules as expected, while either LRO1 is a net TAG consumer under our experimental conditions, moving acyl chains from the acylglycerol population to lyso-phospholipids, or the deletion of LRO1 has increased the overall carbon flux entering lipid biosynthesis.

No shifts in the balances between phosphatidylcholine (PC) and lyso-PC (LPC), or phosphatidylethanolamine (PE) and lyso-PE (LPE) were observed (Fig. 7c), and no other major lipid species were significantly depleted in the LRO1D mutant (Additional file 1: Table S5) suggesting the extra carbon entering the DAG and TAG pools in 


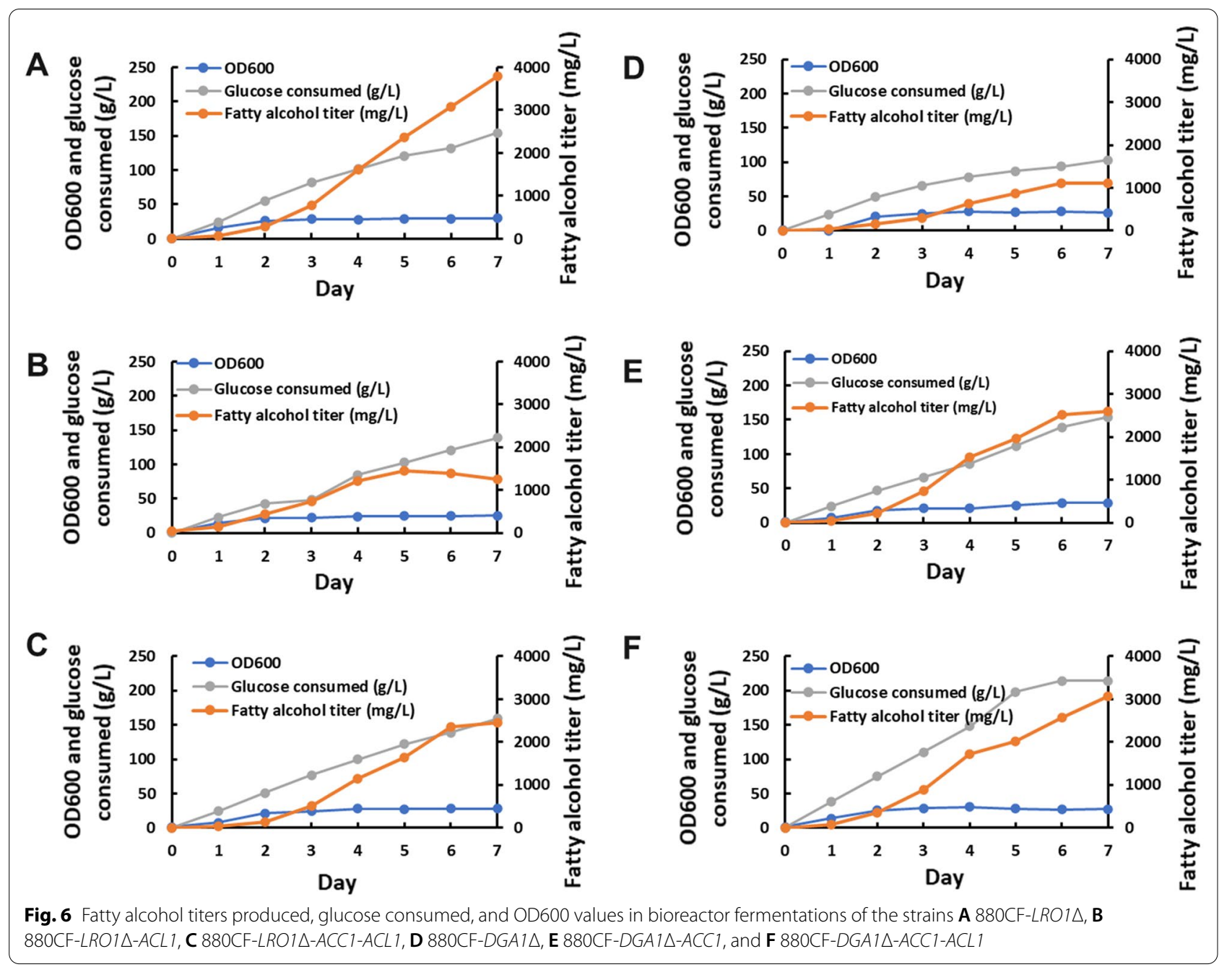

Table 2 Peak fatty alcohol titers and yields resulting from pulsefed batch fermentation in Eppendorf DASbox Mini Bioreactors for 7 days

\begin{tabular}{|c|c|c|}
\hline Genotype & FOH titer (mg/L) & $\begin{array}{l}\mathrm{FOH} \\
\text { yield (g/g } \\
\text { glucose) }\end{array}$ \\
\hline $880 C F-L R 01 \triangle$ & 3.7 & 0.024 \\
\hline $880 C F-L R O 1 \triangle-A C L 1$ & 1.5 & 0.014 \\
\hline $880 C F-L R 01 \triangle-A C C 1-A C L 1$ & 2.7 & 0.016 \\
\hline $880 C F-D G A 1 \triangle$ & 1.1 & 0.011 \\
\hline $880 C F-D G A 1 \triangle-A C C 1$ & 2.7 & 0.017 \\
\hline $880 C F-D G A 1 \triangle-A C C 1-A C L 1$ & 3.1 & 0.014 \\
\hline
\end{tabular}

this mutant must have come from outside the lipid network. A comparison of the carbon consumption of the three strains showed $880 \mathrm{CF}-L R O 1 \Delta$ having the highest glucose consumption after six days of growth, at $14 \mathrm{~g} / \mathrm{L}$ compared to $10.5 \mathrm{~g} / \mathrm{L}$ consumed by $880 \mathrm{CF}$ (Additional file 1: Fig. S1). Therefore, we consider the most likely explanation for the increased fatty alcohol production and DAG/TAG production of $880 \mathrm{CF}-L R O 1 \Delta$ to be a general increase in sugar uptake and carbon flux entering the lipid biosynthesis pathway, although the exact mechanism causing this increase in flux warrants further study. Finally, we also observed that cardiolipin $(\mathrm{CL})$ levels in the LRO1A strain were significantly lowered (Fig. 7d), suggesting disruptions to phosphatidylglycerol metabolism may have occurred as a result of the knockout, although the decrease in this membrane lipid species alone is not enough to account for the much larger increase in TAGs and DAGs.

\section{Discussion}

While $R$. toruloides has been explored as a production host for a variety of molecules, fewer efforts have aimed to engineer its metabolism and improve product titers, 

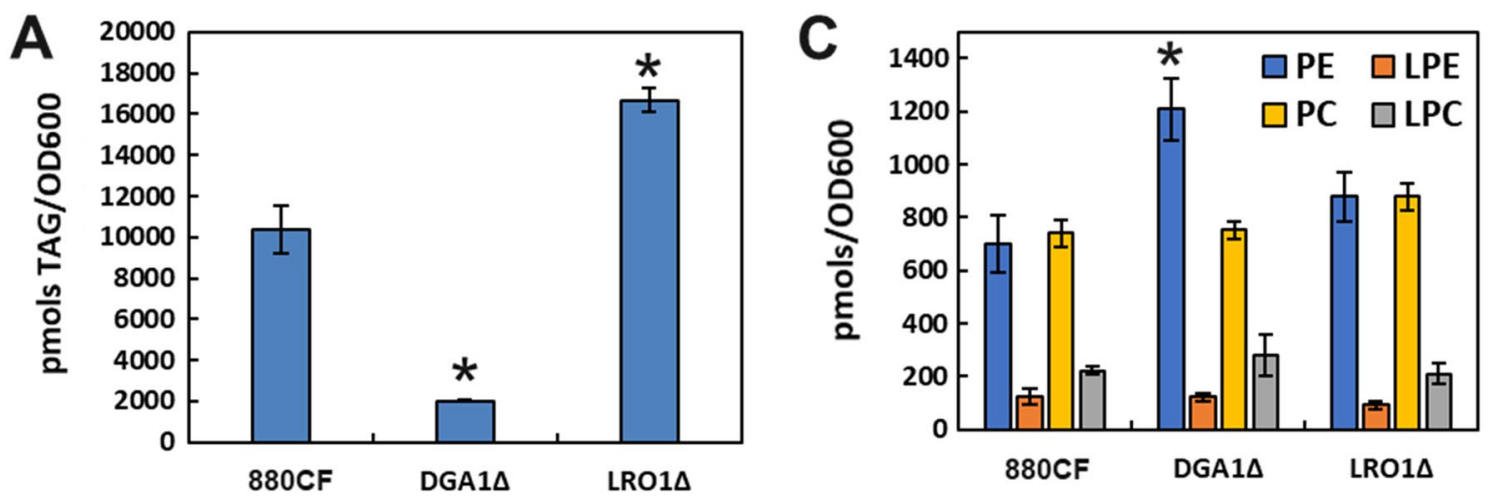

B

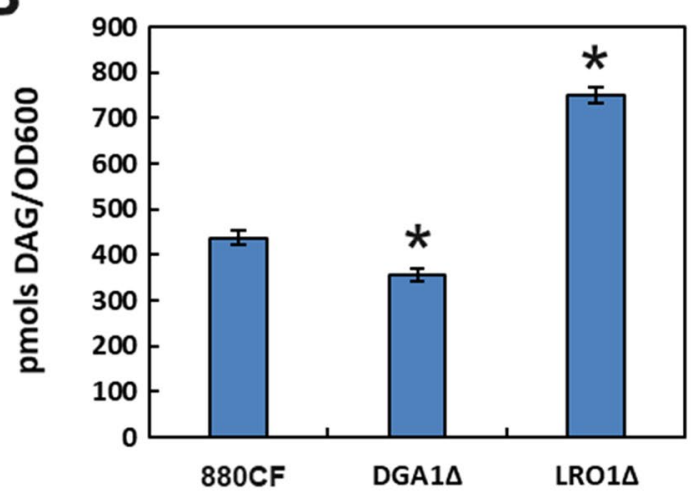

D

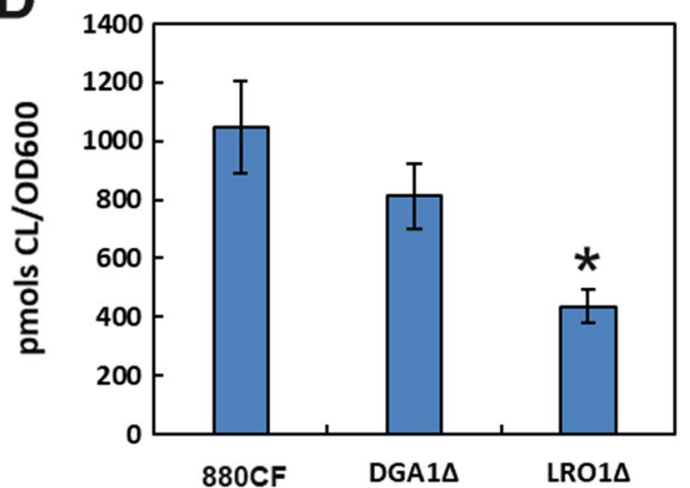

Fig. 7 Intracellular concentrations of the lipid species A TAG; B DAG; C PC, PE, LPC and LPE; and $\mathbf{D} C L$ as determined by lipidomics, in the strains $880 \mathrm{CF}, 880 \mathrm{CF}-D G A 1 \triangle$, and 880CF-LRO1 $\triangle$. Error bars represent \pm standard deviation of biological triplicates. $P E$ phosphatidylethanolamine, $P C$ phosphatidylcholine, LPC lyso-phosphatidylcholine, DAG diacylglyceride, TAG triacylglyceride, LPE lyso-phosphatidylethanolamine, $C L$ cardiolipin. Presence of an asterisk indicates statistically significant improvement compared to 880CF ( $p<0.05$, student $t$-test)

particularly with strategies incorporating the still-difficult process of gene disruption. Zhang and coworkers identified a number of overexpression targets capable of improving lipid production in $R$. toruloides, including $A C C 1, D G A 1, M E 1$, and $S C D 1$, and investigated the knockout of a single gene, PEX10, although its knockout did not increase the lipid titer $[12,25]$. However, numerous $R$. toruloides strains developed to produce other bioproducts such as terpenoids [36-38], fatty acid ethyl esters [39], and, until this study, fatty alcohols [10, 22] have not been optimized further than the expression of the heterologous enzyme responsible for the formation of the final product. Therefore, the development of a workflow for performing sophisticated metabolic engineering as well as the identification of broadly useful metabolic engineering targets in this non-model yeast is of significant importance to the research community.

In this study, a CRISPR/Cas9 tool based on previous reports $[15,16]$ was used in conjunction with constitutive gene overexpression to create an array of single, double, and triple overexpression/deletion mutants in a fatty alcohol-producing strain derived from IFO0880. Notably, the use of CRISPR/Cas9 for the generation of knockouts greatly improved the throughput of the $R$. toruloides strain engineering process, allowing the efficient creation of eight knockout strains for characterization. In screening the engineered strains, we found that increasing the supply of precursor metabolites in the form of acetyl-CoA and malonyl-CoA through overexpression of $A C L 1$ and $A C C 1$ and reducing competition for fatty acyl-CoA molecules from the lipid biosynthetic pathway through disruption of DGA1 and LRO1 proved to be the most effective strategies for improving the fatty alcohol titer. Increasing the production of the reducing cofactor $\mathrm{NADPH}$, used for acyl-CoA biosynthesis and the reduction of acyl chains to fatty alcohols, was less effective, suggesting NADPH supply may not be limiting for this product in $R$. toruloides. Disruption fatty alcohol oxidaselike genes (identified based on homology to $Y$. lipolytica FAO1) and peroxisomal transporter $P X A 1$-like genes had no effect, as did overexpression of SCD1, AMPD1, and $F A A 1+T G L 2$, and disruption of ARE1. 
While $L R O 1$ and DGA1 were selected as knockout targets based on similar reasoning (attempting to increase availability of acyl-CoA chains by preventing their incorporation into TAGs), the significantly differing properties of the resulting knockout strains highlight the different metabolic roles played by these enzymes in $R$. toruloides. Most notably, while the DGA1D mutant had largely abolished TAG formation compared to the wild-type strain, the LRO1D mutant surprisingly had elevated DAG and TAG levels. This stands in contrast to $Y$. lipolytica, where $D G A 1 \triangle$ and $L R O 1 \triangle$ were found to moderately lower TAG levels individually and a DGA1 $L R O 1 \Delta$ double mutant showed a more substantial decrease in TAG [30]. An additional report in $Y$. lipolytica demonstrated that in this yeast species, overexpression of $L R O 1$ increased lipid production [40]. Our finding in $R$. toruloides implicates $D G A 1$ as the major producer of TAGs, while the role of $L R O 1$ is less certain. For future studies of lipid production in $R$. toruloides, both overexpression and knockout of LRO1 may be informative to explore. The fact that the LRO1 $\triangle$ mutant showed a greater improvement in fatty alcohol production than the $D G A 1 \Delta$ mutant is partially explained by the increased glucose consumption

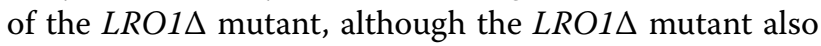
showed a higher carbon yield in culture tube and bioreactor fermentations.

Combining the beneficial overexpression of ACL1 and $A C C 1$ was found to further increase the fatty alcohol titer in the DGA1 $\triangle$ strain background, up to $3.1 \mathrm{~g} / \mathrm{L}$ in bioreactor fermentation, while the $L R O 1 \triangle$ mutant's titer of $3.7 \mathrm{~g} / \mathrm{L}$ in the bioreactor was not further improved. This discrepancy may be due to different characteristics of the starting strains, with $880 \mathrm{CF}-D G A 1 \triangle$ producing about half as much fatty alcohol as $880 \mathrm{CF}-L R O 1 \Delta$. As both of these knockouts are reasoned to improve the fatty alcohol production by redirecting fatty acyl-CoA flux from TAG formation to fatty alcohol formation, 880CF- LRO1D likely has a higher level of fatty acyl-CoA available as substrate for the FAR. Overexpression of $A C C 1$ and $A C L 1$ is intended to increase production of fatty acyl-CoA, but availability of this precursor may have been a limiting factor for fatty alcohol production only in 880CF-DGA1 .

Cordova and coworkers were able to produce $5.8 \mathrm{~g} / \mathrm{L}$ fatty alcohols in a $Y$. lipolytica strain lacking $\beta$-oxidation $(M F E 1 \triangle)$ and peroxisome biogenesis (PEX10D), but containing DGA1 overexpression [9]. While disruption of peroxisomal transporters was ineffective in our study and a PEX10D mutant could not be obtained (the gene has been reported to be essential in IFO0880 [27]), in the future a more comprehensive elimination of $\beta$-oxidation, such as by deletion of MFE1, may further improve fatty alcohol production. The high fatty alcohol titer in a DGA1-overexpressing $Y$. lipolytica strain is interesting, but it may be that $D G A 1 \Delta$ rather than overexpression would further improve the titer of fatty alcohols as strains with native $D G A 1$ expression levels or $D G A 1 \triangle$ were not compared. In our study and D'Espaux and coworkers' study in S. cerevisiae, DGA1 $\triangle$ improved fatty alcohol production [14]. Also in agreement between these studies is the beneficial effect of overexpression of $A C C 1$, while upregulation of unsaturated fatty-acyl production to potentially relieve feedback inhibition of saturated fatty acyl-CoA on the fatty acid synthase complex [1] was more beneficial in S. cerevisiae (OLE1 overexpression) than $R$. toruloides (SCD1 overexpression), resulting in a fourfold increase in fatty alcohol titer compared to the parent strain. In contrast, $L R O 1 \Delta$ was effective in promoting $R$. toruloides's fatty alcohol production but detrimental to $S$. cerevisiae's. The difference in response to SCD1 overexpression may be attributable to the different fatty acid profiles of $S$. cerevisiae and $R$. toruloides: $S$. cerevisiae produces predominantly saturated C16 fatty acids and alcohols, while saturated and unsaturated $\mathrm{C} 18$ fatty acids and alcohols are major components of the $R$. toruloides product spectrum $[10,14]$. This difference could confound the effect of $S C D 1$ overexpression in numerous ways, such as through additional post-translational regulation of the SCD1 protein, or through the presence of much greater amounts of saturated C18-CoA substrate for the enzyme to act on to meaningfully shift the fatty acid profile.

Production of fatty alcohols has been previously reported in $R$. toruloides through heterologous expression of MaFAR without further engineering, with $8 \mathrm{~g} / \mathrm{L}$ achieved in a bioreactor on YP-sucrose with $5 \mathrm{~g} / \mathrm{L}$ tergitol and no organic overlay [10] and $1.7 \mathrm{~g} / \mathrm{L}$ achieved in a bioreactor on synthetic complete media with $0.1 \mathrm{~g} / \mathrm{L}$ of the nonionic surfactant tergitol and a $20 \% \mathrm{vol} / \mathrm{vol}$ dodecane overlay to improve fatty alcohol extraction [22]. This study, in comparison, achieved a maximum titer of $3.7 \mathrm{~g} / \mathrm{L}$ in a bioreactor on synthetic complete media without tergitol and with a $20 \% \mathrm{vol} / \mathrm{vol}$ dodecane overlay in a $L R O 1 \triangle$ strain. Improved titers were also identified in DGA1 $\triangle$ as well as $A C C 1$ and $A C L 1$ overexpressing strains identifying all of these targets as well as $L R O 1 \triangle$ as relevant to the fatty alcohol overproduction phenotype in $R$. toruloides. Combination of these metabolic engineering targets with other strategies such as addition of nonionic surfactants or different media formulations may enable additional increases in fatty alcohol productivity from $R$. toruloides. Furthermore, as the gene editing toolkit for $R$. toruloides becomes more advanced, the use of selection marker recycling or an episomal plasmid will facilitate the creation of strains with a larger number of modifications and further improved titers. 


\section{Conclusions}

In this study, a Cas9-FAR-expressing strain of $R$. toruloides IFO0880 was created and used as a platform organism for the exploration of 16 overexpression and deletion metabolic engineering targets. Several promising targets were explored combinatorially at the culture tube and bioreactor scale, and the best-performing strain, harboring $L R O 1 \Delta$, produced $3.7 \mathrm{~g} / \mathrm{L}$ fatty alcohols from synthetic media. In contrast to findings in other yeast, DGA1 and LRO1 knockouts were both found to increase fatty alcohol production in $R$. toruloides, while a lipidomic survey showed that DGA1 is the predominant TAG-producing protein in this yeast. Based on these findings, the role of LRO1 in $R$. toruloides lipid biosynthesis and fatty acyl-CoA consumption appears to differ from other yeasts. Further investigation may lead to new insights about the lipid metabolism of $R$. toruloides.

\section{Methods}

\section{Strains and media}

The $R$. toruloides strain IFO0880 was grown at $30{ }^{\circ} \mathrm{C}$, $250 \mathrm{rpm}$. YPD media (10 g/L yeast extract, $20 \mathrm{~g} / \mathrm{L}$ peptone, $20 \mathrm{~g} / \mathrm{L}$ glucose) was used for routine handling of cells. For selection or maintenance of transformants, $200 \mu \mathrm{g} / \mathrm{mL}$ G418 (KSE Scientific, Durham, NC), $100 \mu \mathrm{g} / \mathrm{mL}$ nourseothricin (Gold Biotechnology, St. Louis, MO), or $50 \mu \mathrm{g} / \mathrm{mL}$ hygromycin (Gold Biotechnology, St. Louis, MO) was supplemented. Fatty alcohol production was measured in synthetic complete medium (1.7 g/L yeast nitrogen base (BD, Franklin, NJ), $5 \mathrm{~g} / \mathrm{L}$ ammonium sulfate, $0.78 \mathrm{~g} / \mathrm{L}$ complete synthetic mixture (MP Biomedicals, Santa Ana, CA), 40 g/L glucose) adjusted to $\mathrm{pH} 7$ using sodium hydroxide. Culture tube-scale fatty alcohol fermentation experiments were performed by preculturing the yeast for $48 \mathrm{~h}$ in $\mathrm{SC}$ media, then inoculating $5 \mathrm{~mL}$ of SC media in glass culture tubes to an OD600 of 0.1 , with a $10 \%$ dodecane overlay containing $100 \mathrm{mg} / \mathrm{L}$ of pentadecane as internal standard and grown for 6 days.

Standard cloning was performed in the Escherichia coli strain NEB10 $\beta$ (New England Biolabs, Ipswich, MA). Cells were grown on Luria Broth (LB) medium at $37{ }^{\circ} \mathrm{C}, 250 \mathrm{rpm}$ with $100 \mu \mathrm{g} / \mathrm{mL}$ ampicillin or $50 \mu \mathrm{g} / \mathrm{mL}$ kanamycin. Multi-fragment cloning of $20 \mathrm{~kb}$ and larger plasmids was performed in S. cerevisiae BY4741 using DNA assembler [41], with growth on YPD or synthetic uracil dropout media $(1.7 \mathrm{~g} / \mathrm{L}$ yeast nitrogen base, $5 \mathrm{~g} / \mathrm{L}$ ammonium sulfate, $0.78 \mathrm{~g} / \mathrm{L}$ complete synthetic mixture without uracil, $20 \mathrm{~g} / \mathrm{L}$ glucose).

\section{Gene synthesis}

Genes were synthesized by Twist Bioscience (San Francisco, CA) following codon optimization by the JGI BOOST tool, set to use the most frequent codon for each amino acid as they occur in $R$. toruloides [42].

\section{Plasmid construction}

The plasmid pRTN was constructed for heterologous expression or native gene overexpression from the E. coli elements of pUC19 (pMB1 origin, ampicillin resistance), the $S$. cerevisiae elements of pRS426 $(2 \mu$ origin and $U R A 3$ selection marker), the strong $R$. toruloides $\mathrm{p} 17$ promoter, GFP, the T35S terminator, and a $R$. toruloides nourseothricin resistance cassette from pGI2 [25] using DNA assembler. The promoter was subsequently replaced if needed using $A f l \mathrm{II}$ and $M f e I$ digestion, and expressed gene was replaced using $M f e I$ and SpeI digestion. Promoters and native genes were PCR amplified from IFO0880 genomic DNA extracted using lithium acetate/ SDS/heat lysis [43], while heterologous genes were synthesized by Twist Bioscience. Multi-gene expression plasmids were pieced together from either the pRTN or NM9 [15] backbones (for nourseothricin or G418 resistance, respectively) and the appropriate promoter and gene elements using DNA assembler. To prevent complications in the assembly, unique terminators (Tnos, T35S, and Ttub) were used for each gene and the selection marker.

The plasmid pRTH was constructed for gRNA cloning and expression from the E. coli elements of pUC19 (pMB1 origin, ampicillin resistance), the $S$. cerevisiae elements of pRS426 ( $2 \mu$ origin and URA3), a gRNA expression cassette with the IFO0880 5S rRNA, tRNA ${ }^{\text {Tyr }}, 2$ BsaI sites, the $S$. cerevisiae SUP4 terminator, and a $R$. toruloides hygromycin resistance cassette from pZPK-PGPDHYG-Tnos [44] using DNA assembler. gRNAs were cloned by digesting pRTH with $B s a \mathrm{I}$ and ligating two annealed and 5'-phosphorylated oligos with a forward strand 5' GGGA and reverse strand 5' AAAC overhang. For attempted double deletions, 2 tandem gRNA expression cassettes were synthesized as a gBLCK and cloned into the same BsaI restriction sites using Golden Gate assembly. A list of primers used in this study is provided in Additional file 1: Table S6, and a list of plasmids created is provided in Additional file 1: Table S7.

\section{Transformation of $\boldsymbol{R}$. toruloides}

$R$. toruloides was transformed using heat shock as has been described previously [16]. Briefly, a colony was picked and cultured overnight in YPD supplemented with an appropriate antibiotic if necessary. The overnight culture was used to inoculate a shake flask with 25 or $50 \mathrm{~mL}$ of YPD (for up to 5 or 10 transformations, respectively) 
to an OD600 of 0.2 and cultured for $4 \mathrm{~h}$. The cells were collected by centrifugation, washed with water twice, and mixed with $240 \mu \mathrm{L}$ PEG3350 (Sigma Aldrich, St. Louis, $\mathrm{MO}$ ), $36 \mu \mathrm{L} 1 \mathrm{M}$ lithium acetate (Sigma Aldrich, St. Louis, $\mathrm{MO}$ ), $50 \mu \mathrm{L}$ of $2 \mathrm{mg} / \mathrm{mL}$ salmon sperm DNA (Sigma Aldrich, St. Louis, MO), and 1-2 $\mu \mathrm{g}$ of linear DNA dissolved in $40 \mu \mathrm{L}$ of water. The cells were incubated with shaking in the transformation mixture for $30 \mathrm{~min}$ at $30{ }^{\circ} \mathrm{C}$. Then, $34 \mu \mathrm{L}$ of DMSO was added to the mixture, which was briefly vortexed, and heat shocked at $42{ }^{\circ} \mathrm{C}$ for $15 \mathrm{~min}$. The cells were collected, washed once with water, resuspended in $2 \mathrm{~mL}$ YPD, and allowed to recover overnight. The cells were then collected and spread to YPD agar plates supplemented with the appropriate antibiotic. Transformation efficiencies of $\sim 10^{2} \mathrm{CFU} / \mu \mathrm{g}$ DNA were typically observed.

\section{Genetic manipulation of $R$. toruloides}

All genetic modifications to $R$. toruloides were made by random integration of linear PCR or restriction digestion fragments using heat shock transformation. After cloning of the gene or gRNA expression cassette to be transformed to a plasmid as described above, fragments were prepared either through PCR amplification (with the primers $Z P K F / R$, or gRNA $F / R$, respectively) from their plasmid, or (for cassettes longer than $7 \mathrm{~kb}$ ) through excision of this fragment by digestion with suitable restriction enzymes, followed by spin column purification. Overexpression cassettes were created by expressing either the $R$. toruloides genomic copy of a gene (for endogenous genes) or a synthetic codon optimized gene (for heterologous genes) with the strong $p 17$ promoter. Deletion mutants were created by transforming a single gRNA targeting the first $10 \%$ of the target gene ORF to generate frame shift mutations following NHEJ DNA repair. gRNAs were designed using the Benchling gRNA tool. For verification of deletion mutants, genomic DNA was extracted using lithium acetate/SDS/heat lysis [43], PCR amplified at the target locus and sequenced. For gene activation targets, genomic DNA was extracted using the same method and integration was verified using colony PCR.

\section{Bioreactor fermentation}

For bioreactor experiments, a seed culture was grown for $48 \mathrm{~h}$ in a culture tube in SC media, then transferred entirely to $50 \mathrm{~mL}$ of media in a shake flask for another $24 \mathrm{~h}$. The cells were collected, washed once with water, and used to inoculate $100 \mathrm{~mL}$ of SC media to an OD600 of 1 in $250 \mathrm{~mL}$ Eppendorf DASbox Mini Bioreactors (Eppendorf, Hamburg, Germany) with $20 \mathrm{~mL}$ of dodecane containing $100 \mathrm{mg} / \mathrm{L}$ pentadecane. Bioreactors were set to maintain a $\mathrm{pH}$ of 7 , agitation of $1200 \mathrm{rpm}$, temperature of $30^{\circ} \mathrm{C}$, and air flow rate of 6 standard liters per minute. Antifoam 204 was added to control foaming as necessary (typically 1 drop per reactor). Each day, samples were collected to measure OD600, fatty alcohol titer, and glucose concentration. Glucose was added to restore the concentration to $50 \mathrm{~g} / \mathrm{L}$ for the first five days.

\section{Analytical methods}

Culture tube and bioreactor fermentation experiments were performed with a $10 \%$ or $20 \%$ dodecane overlay, respectively, with $100 \mathrm{mg} / \mathrm{L}$ pentadecane added as an internal standard. For quantification of fatty alcohols, $200 \mu \mathrm{L}$ or $1 \mathrm{~mL}$ (for culture tube and bioreactor, respectively) was removed and centrifuged to separate the dodecane layer. $10 \mu \mathrm{L}$ of dodecane was mixed with $90 \mu \mathrm{L}$ of ethyl acetate and analyzed on an Agilent 8860 GC-FID with a 30-m DB-5 column (Agilent, Santa Clara, CA) with a temperature ramp of $70{ }^{\circ} \mathrm{C}$ for $3 \mathrm{~min}$, increase at $20{ }^{\circ} \mathrm{C} /$ minute to $320{ }^{\circ} \mathrm{C}$, hold $320{ }^{\circ} \mathrm{C}$ for $1 \mathrm{~min}$. The total fatty alcohol titer was calculated as the sum of 1-hexadecanol, 1-octadecanol, and oleyl alcohol titers.

Glucose consumption was measured using an Agilent 1260 Infinity HPLC with a refractive index detector (RID) and $\mathrm{H}^{+}$column (Rezex ROA-Organic Acid; Phenomenex, Torrance, $\mathrm{CA}$ ). The column and detector were run at $50{ }^{\circ} \mathrm{C}$ and $0.6 \mathrm{~mL} / \mathrm{min}$ of $0.005 \mathrm{~N} \mathrm{H}_{2} \mathrm{SO}_{4}$ was used as the mobile phase.

\section{Lipidomics}

Cells were cultured in pH7-adjusted SCD media with a $10 \%$ dodecane overlay in glass culture tubes under the same conditions as for the fatty alcohol fermentations and cell pellets were collected after 6 days of growth following inoculation. Lipidomics analysis of both the reference and mutant yeast strains was performed using a two-step chloroform-methanol extraction as described elsewhere $[45,46]$. Briefly, harvested cells were washed with $150 \mathrm{mM}$ ammonium bicarbonate $(\mathrm{ABC})$ followed by cell lysis with zirconium glass beads. Lipids were extracted from 1 OD unit of cell lysate, to which an internal lipid standard mix was added, in a 2-step method. First extraction was performed with $1 \mathrm{~mL}$ of 15:1 chloroform-methanol after which the chloroform layer was aspirated, dried, and then resuspended in an infusion solvent. The second extraction was performed on the remaining aqueous layer with $1 \mathrm{~mL}$ of 2:1 chloroformmethanol and again the chloroform layer was aspirated, dried, and resuspended in an infusion solvent. Both resuspended extracts were then infused into a Q-Exactive mass spectrometer via the Advion Triversa Nanomate in the nano-electrospray mode. The infusion solvent used for the dried lipid extract from the 15:1 extraction contained $7.5 \mathrm{mM}$ ammonium formate in a mix of 
chloroform-methanol-propanol 1:2:4 (v/v) whereas the infusion solvent for the dried extract from the 2:1 extraction contained $0.05 \%$ methylamine in a mix of chloroform-methanol 1:5 (v/v) [46].

Identification and quantification of lipid species and classes from the lipidomic data generated by the mass spectrometer was performed using an in-house Python script that employed the pymzml library. Raw data files were input to the pipeline after conversion to the open source mzML format. The data analysis pipeline consisted of scan averaging, offline calibration, deisotoping and identification, quantification, and quantification across replicates. The list of internal standards and their absolute amounts used for spike-in are listed in Additional file 1: Table S8. The code used for analysis is available upon request.

\section{Supplementary Information}

The online version contains supplementary material available at https://doi. org/10.1186/s12934-022-01750-3.

Additional file 1. Figure S1. Glucose consumption of 880CF, $880 C F-D G A 1 \triangle$ and $880 C F-L R O 1 \triangle$. Table S1. DNA sequences of heterologous, codon-optimized genes evaluated in this study. Table S2. List of gRNAs used in this study. Table S3. List of Mycocosm proteinIDs for R. toruloides IFO0880 gene targets in this study. Table S4. List of R. toruloides strains used in this study. Table $\mathbf{S 5}$. Results of lipidomic comparison of $880 C F, 880 C F-D G A 1 \triangle$, and $880 C F-L R O 1 \triangle$. Table S6. List of primers used in this study. Table S7. List of plasmids used in this study. Table $\mathbf{8 8 .}$ List of lipidomics internal standards and their absolute amounts used for spike-in.

\section{Acknowledgements}

We thank Christopher Rao for the gifts of the strain IFO0880 and the plasmids pGI2 and pGI2_880_ACC.

\section{Funding}

This work was funded by the DOE Center for Advanced Bioenergy and Bioproducts Innovation (U.S. Department of Energy, Office of Science, Office of Biological and Environmental Research under Award Number DE-SC0018420). Any opinions, findings, and conclusions or recommendations expressed in this publication are those of the author(s) and do not necessarily reflect the views of the U.S. Department of Energy.

\section{Availability of data and materials}

All data and materials produced in this study are available upon reasonable request.

\section{Declarations}

Ethics approval and consent to participate

Not applicable.

\section{Consent for publication}

Not applicable.

\section{Competing interests}

None declared.

\section{Author details}

'Department of Chemical and Biomolecular Engineering, Carl R. Woese Institute for Genomic Biology, University of Illinois at Urbana-Champaign, Urbana,
IL 61801, USA. ${ }^{2}$ U.S. Department of Energy Center for Bioenergy and Bioproducts Innovation (CABBI), Urbana, IL 61801, USA. ${ }^{3}$ Department of Chemical Engineering, Pennsylvania State University, University Park, PA 16802, USA. ${ }^{4}$ Departments of Chemistry, Biochemistry, and Bioengineering, University of Illinois at Urbana-Champaign, Urbana, IL 61801, USA.

Received: 6 September 2021 Accepted: 25 January 2022

Published online: 19 February 2022

\section{References}

1. Krishnan A, McNeil BA, Stuart DT. Biosynthesis of fatty alcohols in engineered microbial cell factories: advances and limitations. Front Bioeng Biotechnol. 2020;8:1385.

2. Noweck K, Grafahrend W. Fatty alcohols. In: Ullmann's encyclopedia of industrial chemistry. Weinheim, Germany: Wiley-VCH Verlag GmbH \& Co. KGaA; 2006.

3. Zimmermann H, Walzl R. Ethylene. In: Ullmann's encyclopedia of industrial chemistry. Weinheim, Germany: Wiley-VCH Verlag GmbH \& Co. KGaA; 2009.

4. Mohd Azhar SH, Abdulla R, Jambo SA, Marbawi H, Gansau JA, Mohd Faik AA, et al. Yeasts in sustainable bioethanol production: a review. Biochem Biophys Rep. 2017;10:52-61.

5. Paddon CJ, Keasling JD. Semi-synthetic artemisinin: a model for the use of synthetic biology in pharmaceutical development. Nat Rev Microbiol. 2014;12(5):355-67.

6. Hong KK, Nielsen J. Metabolic engineering of Saccharomyces cerevisiae: a key cell factory platform for future biorefineries. Cell Mol Life Sci. 2012;69(16):2671-90.

7. Fatma Z, Jawed K, Mattam AJ, Yazdani SS. Identification of long chain specific aldehyde reductase and its use in enhanced fatty alcohol production in E. coli. Metab Eng. 2016;37:35-45.

8. Kaczmarzyk D, Cengic I, Yao L, Hudson EP. Diversion of the long-chain acyl-ACP pool in Synechocystis to fatty alcohols through CRISPRi repression of the essential phosphate acyltransferase PIsX. Metab Eng. 2018:45:59-66.

9. Cordova LT, Butler J, Alper HS. Direct production of fatty alcohols from glucose using engineered strains of Yarrowia lipolytica. Metab Eng Commun. 2020;10: e00105.

10. Fillet S, Gibert J, Suárez B, Lara A, Ronchel C, Adrio JL. Fatty alcohols production by oleaginous yeast. J Ind Microbiol Biotechnol. 2015;42(11):1463-72.

11. Yu T, Zhou YJ, Huang M, Liu Q, Pereira R, David F, et al. Reprogramming yeast metabolism from alcoholic fermentation to lipogenesis. Cell. 2018:174(6):1549-1558.e14.

12. Zhang $S$, Ito M, Skerker JM, Arkin AP, Rao CV. Metabolic engineering of the oleaginous yeast Rhodosporidium toruloides IFO0880 for lipid overproduction during high-density fermentation. Appl Microbiol Biotechnol. 2016;100(21):9393-405.

13. Liu Y, Chen S, Chen J, Zhou J, Wang Y, Yang M, et al. High production of fatty alcohols in Escherichia coli with fatty acid starvation. Microb Cell. Fact. 2016;15(1):129.

14. d'Espaux L, Ghosh A, Runguphan W, Wehrs M, Xu F, Konzock O, et al. Engineering high-level production of fatty alcohols by Saccharomyces cerevisiae from lignocellulosic feedstocks. Metab Eng. 2017;42:115-25.

15. Schultz JC, Cao M, Zhao H. Development of a CRISPR/Cas9 system for high efficiency multiplexed gene deletion in Rhodosporidium toruloides. Biotechnol Bioeng. 2019;116(8):2103-9.

16. Otoupal PB, Ito M, Arkin AP, Magnuson JK, Gladden JM, Skerker JM. Multiplexed CRISPR-Cas9-based genome editing of Rhodosporidium toruloides. mSphere. 2019;4(2):00099-119.

17. Jiao X, Zhang Y, Liu X, Zhang Q, Zhang S, Zhao ZK. Developing a CRISPR/ Cas9 system for genome editing in the basidiomycetous yeast $R$ hodosporidium toruloides. Biotechnol J. 2019;14(7): e1900036.

18. Liu X, Zhang Y, Liu H, Jiao X, Zhang Q, Zhang S, et al. RNA interference in the oleaginous yeast Rhodosporidium toruloides. FEMS Yeast Res. 2019:19:31.

19. Nora LC, Wehrs M, Kim J, Cheng JF, Tarver A, Simmons BA, et al. A toolset of constitutive promoters for metabolic engineering of Rhodosporidium toruloides. Microb Cell Fact. 2019;18(1):117. 
20. Johns AMB, Love J, Aves SJ. Four inducible promoters for controlled gene expression in the oleaginous yeast Rhodotorula toruloides. Front Microbiol. 2016;7:1666-76.

21. Wang $Y$, Lin $X$, Zhang S, Sun W, Ma S, Zhao ZK. Cloning and evaluation of different constitutive promoters in the oleaginous yeast Rhodosporidium toruloides. Yeast. 2016;33(3):99-106.

22. Liu D, Geiselman GM, Coradetti S, Cheng Y, Kirby J, Prahl J, et al. Exploiting nonionic surfactants to enhance fatty alcohol production in Rhodosporidium toruloides. Biotechnol Bioeng. 2020;117(5):1418-25.

23. Willis RM, Wahlen BD, Seefeldt LC, Barney BM. Characterization of a fatty acyl-CoA reductase from Marinobacter aquaeolei VT8: a bacterial enzyme catalyzing the reduction of fatty acyl-CoA to fatty alcohol. Biochemistry. 2011:50(48):10550-8.

24. Partow S, Siewers V, Bjørn S, Nielsen J, Maury J. Characterization of different promoters for designing a new expression vector in Saccharomyces cerevisiae. Yeast. 2010;27(11):955-64.

25. Zhang S, Skerker JM, Rutter CD, Maurer MJ, Arkin AP, Rao CV. Engineering Rhodosporidium toruloides for increased lipid production. Biotechno Bioeng. 2016;113:1056-66.

26. Dinh HV, Suthers PF, Chan SHJ, Shen Y, Xiao T, Deewan A, et al. A comprehensive genome-scale model for Rhodosporidium toruloides IFO0880 accounting for functional genomics and phenotypic data. Metab Eng Commun. 2019;9: e00101.

27. Coradetti ST, Pinel D, Geiselman GM, Ito M, Mondo SJ, Reilly MC, et al. Functional genomics of lipid metabolism in the oleaginous yeast Rhodosporidium toruloides. Elife. 2018;7: e32110.

28. Kim J, Coradetti ST, Kim YM, Gao Y, Yaegashi J, Zucker JD, et al. Multi-omics driven metabolic network reconstruction and analysis of lignocellulosic carbon utilization in Rhodosporidium toruloides. Front Bioeng Biotechnol. 2021;8: 612832.

29. Li Y, Zhao Z, Bai F. High-density cultivation of oleaginous yeast Rhodosporidium toruloides Y4 in fed-batch culture. Enzyme Microb Technol. 2007:41:312-7.

30. Athenstaedt K. YALIOE32769g (DGA1) and YALIOE16797g (LRO1) encode major triacylglycerol synthases of the oleaginous yeast Yarrowia lipolytica. Biochim Biophys Acta Mol Cell Biol Lipids. 1811;10:587-96.

31. Yang $H$, Bard M, Bruner DA, Gleeson A, Deckelbaum RJ, Aljinovic G, et al. Sterol esterification in yeast: a two-gene process. Science (80-). 1996;272(5266):1353-6.

32. Xue Z, Sharpe PL, Hong SP, Yadav NS, Xie D, Short DR, et al. Production of omega-3 eicosapentaenoic acid by metabolic engineering of Yarrowia lipolytica. Nat Biotechnol. 2013:31(8):734-40.

33. Blazeck J, Hill A, Liu L, Knight R, Miller J, Pan A, et al. Harnessing Yarrowia lipolytica lipogenesis to create a platform for lipid and biofuel production. Nat Commun. 2014;5(1):1-10.

34. Qiao K, Imam Abidi SH, Liu H, Zhang H, Chakraborty S, Watson N, et al. Engineering lipid overproduction in the oleaginous yeast Yarrowia lipolytica. Metab Eng. 2015;29:56-65.

35. Beopoulos A, Cescut J, Haddouche R, Uribelarrea JL, Molina-Jouve C, Nicaud JM. Yarrowia lipolytica as a model for bio-oil production. Prog Lipid Res. 2009;48(6):375-87.

36. Yaegashi J, Kirby J, Ito M, Sun J, Dutta T, Mirsiaghi M, et al. Rhodosporidium toruloides: a new platform organism for conversion of lignocellulose into terpene biofuels and bioproducts. Biotechnol Biofuels. 2017;10(1):1-13.

37. Zhuang X, Kilian O, Monroe E, Ito M, Tran-Gymfi MB, Liu F, et al. Monoterpene production by the carotenogenic yeast Rhodosporidium toruloides. Microb Cell Fact. 2019. https://doi.org/10.1186/s12934-019-1099-8.

38. Kirby J, Geiselman GM, Yaegashi J, Kim J, Zhuang X, Tran-Gyamfi MB, et al. Further engineering of $R$. toruloides for the production of terpenes from lignocellulosic biomass. Biotechnol Biofuels. 2021;14(1):101.

39. Zhang Y, Peng J, Zhao H, Shi S. Engineering oleaginous yeast Rhodotorula toruloides for overproduction of fatty acid ethyl esters. Biotechnol Biofuels. 2021;14(1):115.

40. Amalia L, Zhang YH, Ju YH, Tsai SL. Enhanced lipid production in Yarrowia lipolytica Po1g by over-expressing Iro1 gene under two different promoters. Appl Biochem Biotechnol. 2020:191(1):104-11.

41. Shao Z, Zhao H, Zhao H. DNA assembler, an in vivo genetic method for rapid construction of biochemical pathways. Nucleic Acids Res. 2009;37(2):1-10.
42. Oberortner E, Cheng JF, Hillson NJ, Deutsch S. Streamlining the designto-build transition with build-optimization software tools. ACS Synth Biol. 2016;6(3):485-96

43. Lõoke M, Kristjuhan K, Kristjuhan A. Extraction of genomic DNA from yeasts for PCR-based applications. Biotechniques. 2011;50(5):325-8.

44. Lin X, Wang Y, Zhang S, Zhu Z, Zhou YJ, Yang F, et al. Functional integration of multiple genes into the genome of the oleaginous yeast $R$ Rhodosporidium toruloides. FEMS Yeast Res. 2014;14(4):547-55.

45. Ejsing CS, Sampaio JL, Surendranath V, Duchoslav E, Ekroos K, Klemm RW, et al. Global analysis of the yeast lipidome by quantitative shotgun mass spectrometry. Proc Natl Acad Sci. 2009;106(7):2136-41.

46. Klose C, Tarasov K. Profiling of yeast lipids by shotgun lipidomics. Methods Mol Biol. 2016;1361:309-24.

\section{Publisher's Note}

Springer Nature remains neutral with regard to jurisdictional claims in published maps and institutional affiliations.

Ready to submit your research? Choose BMC and benefit from:

- fast, convenient online submission

- thorough peer review by experienced researchers in your field

- rapid publication on acceptance

- support for research data, including large and complex data types

- gold Open Access which fosters wider collaboration and increased citations

- maximum visibility for your research: over $100 \mathrm{M}$ website views per year

At BMC, research is always in progress.

Learn more biomedcentral.com/submissions 